\title{
NEFRECTOMIA UNILATERAL EM CADELA PARASITADA POR Dioctophyma renale : RELATO DE CASO
}

\author{
HERMETO, Larissa Correa ${ }^{1}$ \\ MAUAD, Juliana Rosa Carrijo ${ }^{2}$ \\ MATTEI, Douglas Rodrigo ${ }^{3}$ \\ FERRAREZI, Ana Paula Ferreira ${ }^{4}$ \\ VENTURA, Arlene Sobrinho ${ }^{4}$
}

ISSUE DOI: $10.3738 / 1982.2278 .674$

RESUMO: O dioctophyma renale, conhecido como verme gigante renal é um nematóide de ocorrência mundial que parasita os rins, podendo ser encontrado na cavidade peritoneal e em outros órgãos do cão, e em outras espécies de animais domésticos e silvestres, inclusive o homem. Foi atendida uma cadela sem raça definida, apresentando secreção vaginal sanguinolenta persistente, neoformação vaginal, hiporexia e hematúria. Foram solicitados exames complementares, e através da ultrassonografia abdominal foi detectada imagem sugestiva de dioctofimose, a qual confirmou-se com a visualização de ovos característicos de Dioctophyma renale no sedimento urinário. Após o diagnóstico, foi realizado laparotomia exploratória seguido de nefrectomia do rim direito, e após a conduta terapêutica o animal apresentou excelente recuperação. O presente relato ressalta a importância dos exames complementares para o diagnóstico efetivo, o que proporciona recuperação total do animal parasitado.

Palavras-chave: Cães. Dioctofimose Laparotomia exploratória.

SUMMARY: -Dioctophyma renale, known as giant kidney worm, is a nematode of worldwide occurrence that parasitizes the kidneys and can be found not only in the peritoneal cavity but also in other organs of a dog and in other species of domestic and wild animals, as well as the man. A mongrel bitch presenting with persistent bloody vaginal discharge, vaginal neoformation, appetite loss, and hematuria was treated. Additional tests were requested, and an image suggestive of dioctophymosis was detected through abdominal ultrasound. It was later confirmed when characteristic eggs of Dioctophyma renale were observed in the urinary sediment. After the diagnosis, exploratory laparotomy was performed followed by nephrectomy of the right kidney. The animal had an excellent recovery after such therapy. This report highlights the importance of additional tests for the effective diagnosis, which leads to total recovery of the parasitized animal.

Key words: Dogs. Dioctophymosis. Exploratory laparotomy.

\section{INTRODUÇÃO}

O Dioctophyma renale (GOEZE, 1782), é um parasito conhecido como verme gigante renal, também denominado estrôngilo gigante, Ascaris renalis, Ascaris visceralis, Strongylus gigas ou Eustrongylus gigas, é o maior nematóide conhecido citado na literatura (Urquhart et al., 1998). Possui de 14 a $100 \mathrm{~cm}$ de comprimento por 0,4 a $1,2 \mathrm{~cm}$ de largura e pertence à ordem Enoplida, família Dioctophymatidae (BARRIGA, 2002). Apresenta distribuição mundial e acomete diversas espécies animais, inclusive o homem (OSBORNE et al., 1969). Nos carnívoros, é comumente encontrado nos rins, principalmente o direito, e livre na cavidade abdominal (OSBORNE et al 1969; GARGILI et al., 2002). A epidemiologia deste parasito envolve um ciclo evolutivo complexo onde seus ovos contendo larvas de primeiro estádio são ingeridos por um anelídeo oligoqueta aquático (Lumbriculus variegatus) e o hospedeiro definitivo é infectado a partir de ingestão desses anelídeos ou hospedeiros paratênicos (peixes e rãs) infectados (KOMMERS et al., 1999). O peixe ou a rã ingere o crustáceo com o anelídeo contendo a

\footnotetext{
${ }^{1}$ Doutoranda- Universidade Estadual Júlio de Mesquita Filho- campus Jaboticabal

${ }^{2}$ Professora Doutora Universidade Federal da Grande Dourados

${ }^{3}$ Professor Faculdade Anhanguera de Dourados

${ }^{4}$ Acadêmico Faculdade Anhanguera de Dourados
} 
larva, que ficará encapsulada na musculatura destes hospedeiros paratênicos, portanto, os animais e o homem adquirem o nematóide principalmente a partir da ingestão de carne de peixe ou rã pouco cozida e de anelídeos aquáticos infectados com a forma larval (OSBORNE et al, 1969; BROWN; PRESTWOOD,1988). Na patogenia o efeito final da infecção é a destruição do rim, pois o parênquima é destruído, deixando apenas a cápsula como uma bolsa contendo os ovos; embora possa haver três ou quatro vermes num rim, às vezes há apenas um. Raramente, os vermes podem ocorrer na cavidade abdominal e no tecido conjuntivo subcutâneo. Sinais clínico como apatia, inapetência e emagrecimento podem estar associados a dioctofimose, apesar do curso da parasitose ser muitas vezes assintomático nos animais e pessoas infectadas (URQUART et al.,1998). Os animais afetados podem apresentam hematúria, devido à lesão renal, onde ocorre destruição total do parênquima deste órgão e hipertrofia compensatória do rim contralateral (COSTA et al.,2004). O diagnóstico da presença do helminto pode ser feito pelo achado de ovos operculados de casca espessa e rugosa na urina ou por ultrassonografia dos rins e cavidade abdominal (Barriga, 2002). Nenhuma terapia médica é efetiva (Forrester, 2003) e o tratamento indicado é a nefrectomia ou a nefrotomia, dependendo da gravidade da lesão renal e da presença do nematódeo em um ou ambos os rins, mas o tratamento preconizado é a nefrectomia do rim acometido (CHRISTIE; BJORLING, 1998), podendo ser realizada de forma convencional ou laparoscópica conforme descrito por Brun et al., (2002).

A proposta do presente trabalho é relatar o diagnóstico precoce da dioctiofimose, permitindo a recuperação total do paciente.

\section{RELATO DE CASO}

Uma cadela sem raça definida, três anos de idade, $8.4 \mathrm{~kg}$, pelagem preta, deu entrada ao hospital veterinário, apresentando secreção vaginal sanguinolenta persistente e presença de neoformação vaginal há aproximadamente trinta dias. Segundo o proprietário, o animal estava apresentando hiporexia, hematúria, disúria, normodipsia e normoquezia. Ao exame físico geral foram notadas mucosas hipocoradas, tempo de preenchimento capilar de dois segundos, pêlos ressecados, temperatura retal de $38.6^{\circ} \mathrm{C}$, condição corpórea regular, desidratação moderada, discreto desconforto a palpação abdominal, linfonodos sem alterações dignas de nota. A auscultação torácica demonstrou-se limpa, e a percussão, timpânica. Foi observado uma massa vaginal de formato irregular, de tamanho aproximado de $4 \mathrm{~cm}$, indolor, com odor fétido, aderida ao assoalho vaginal, coloração rosada, de consistência friável, com aspecto vegetante, conjuntamente com secreção serosanguinolenta. Foram solicitados exames complementares como: hemograma completo, urinálise, dosagem sérica de creatinina e ALT, ultrassonografia e citologia da massa vaginal por imprint. Os resultados revelaram anemia normocítica normocrômica, leucocitose por neutrofilia, análise sérica de creatinina $0,7 \mathrm{mg} / \mathrm{dl}$ e ALT 50U/L. A citologia vaginal concluiu que tratava-se de tumor venéreo transmissível de padrão linfocitóide. A ultrassonografia revelou parênquima renal esquerdo sem alterações e, em topografia renal direita, presença de imagem capsular repleta por conteúdo anecóico e, imagem tubular, formada por linhas hiperecóicas e hipoecóicas, vesícula urinária apresentando acentuada quantidade de celularidade e sedimento, e demais estruturas abdominais sem alterações ultrassonográficas (Figura 1). 
Figura 1- Imagem ultrassonográfica da topografia renal direita sugerindo parasitismo por D. renale: parasito em corte transversal (seta pontilhada) e corte longitudinal (seta contínua).

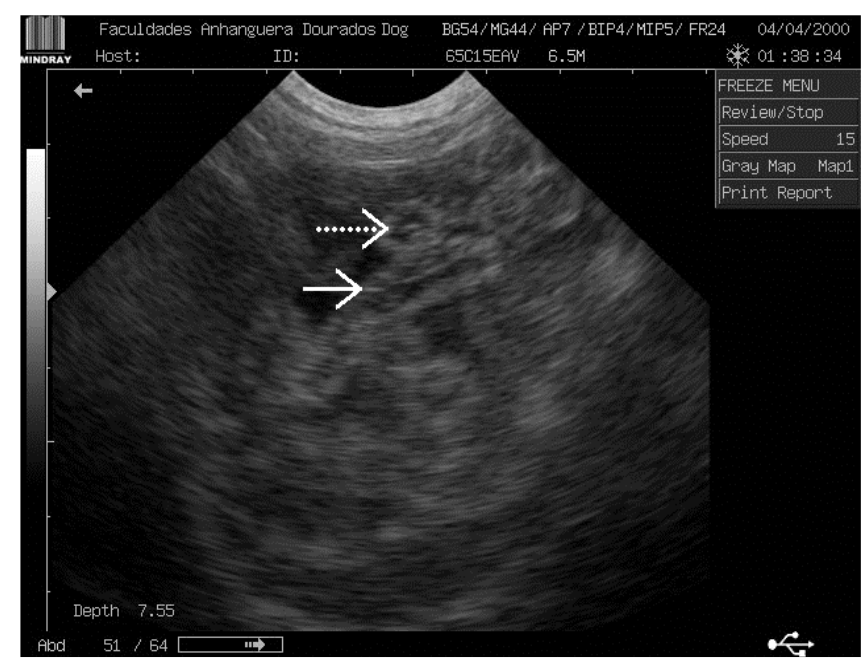

Durante o procedimento ultrassonográfico, foi realizada a cistocentese para a realização de urinálise, na qual foram encontrados após centrifugação e análise do sedimento ovos característicos de Dioctophyma renale (Figura 2), confirmando a suspeita diagnóstica.

Figura 2 - Ovos característicos de D.renale durante avaliação do sedimento urinário.

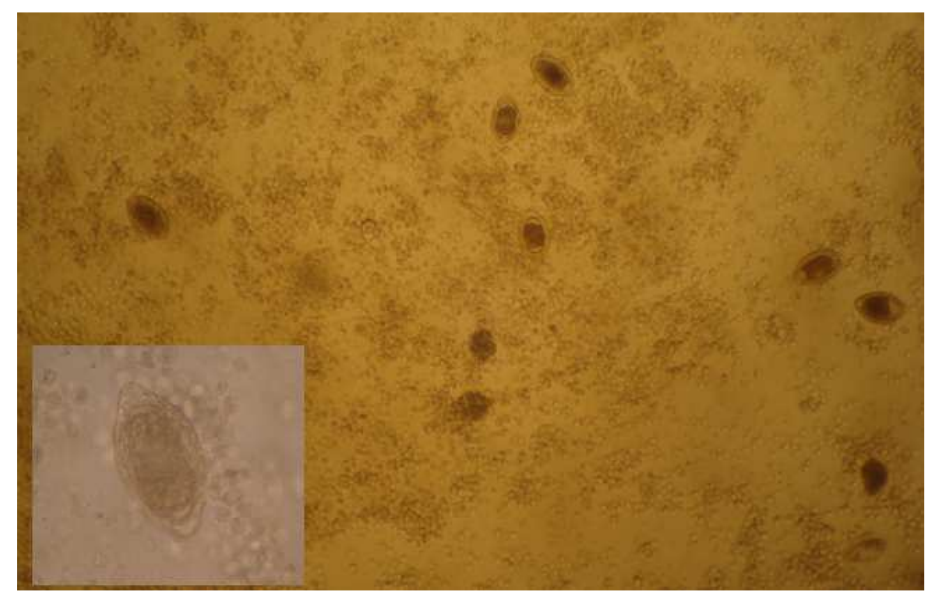

Após o diagnóstico, foi sugerido laparotomia exploratória para a realização da nefrectomia do rim direito. $\mathrm{O}$ animal foi previamente preparado para o procedimento cirúrgico, com jejum alimentar de 12 horas e hídrico de 4 horas. Foi realizada transfusão sanguínea pré-operatória imediata, pois o animal apresentava hematócrito $22 \%$, e o risco de sangramento, hipotensão e hipoxemia durante o transoperatório era uma preocupação pertinente. Foi realizada a medicação pré anestésica com butorfanol $0,2 \mathrm{mg} / \mathrm{kg}$ associado ao midazolam $0,3 \mathrm{mg} / \mathrm{kg}$ por via intramuscular, e aguardado quinze minutos, período em que realizava-se a tricotomia ampla do abdômen. $\mathrm{O}$ animal foi induzido com propofol na dose de $4 \mathrm{mg} / \mathrm{kg}$ intravenoso, intubado, e mantido com a inalação de isofluorano. Antes do início da cirurgia, foi realizado um bólus de amoxicilina com clavulanato de potássio na dose de $12,5 \mathrm{mg} / \mathrm{kg}$. Após a assepsia do campo cirúrgico foi realizada incisão mediana ventral, do processo xifóide a $2 \mathrm{~cm}$ abaixo da cicatriz umbilical. Ao adentrar a cavidade abdominal, foi observado derrame peritoneal, de consistência espessa, coloração amarelo-palha. (Figura 3). 
Figura 3- Derrame peritoneal espesso, amarelo-palha em toda cavidade abdominal.

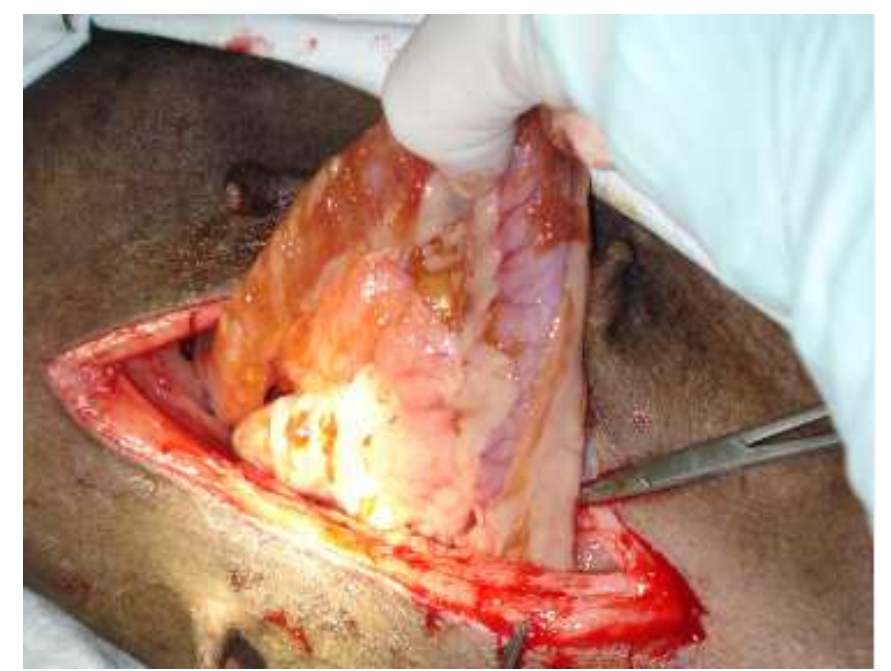

Foi realizada lavagem abdominal abundante com solução fisiológica morna, e primeiramente realizada ovariosalpingohisterectomia de forma rotineira conforme técnica sugerida por Headlund (2008), e seguida de incisão paracostal direita para acesso para nefrectomia do rim direito conforme técnica sugerida por Fossum (2008). Após o término do transoperatório, foi realizada outra lavagem abdominal conforme supra descrito, e finalizado com a síntese das camadas incisionadas. Os materiais cirúrgicos e as luvas cirúrgicas foram trocadas e a nodulectomia da neoformação vaginal foi realizada com episiotomia e ressecção da neoplasia que encontrava-se no assoalho vaginal, conforme sugerido por Klein (2007).

Figura 4- Aspecto final no pós operatório imediato.

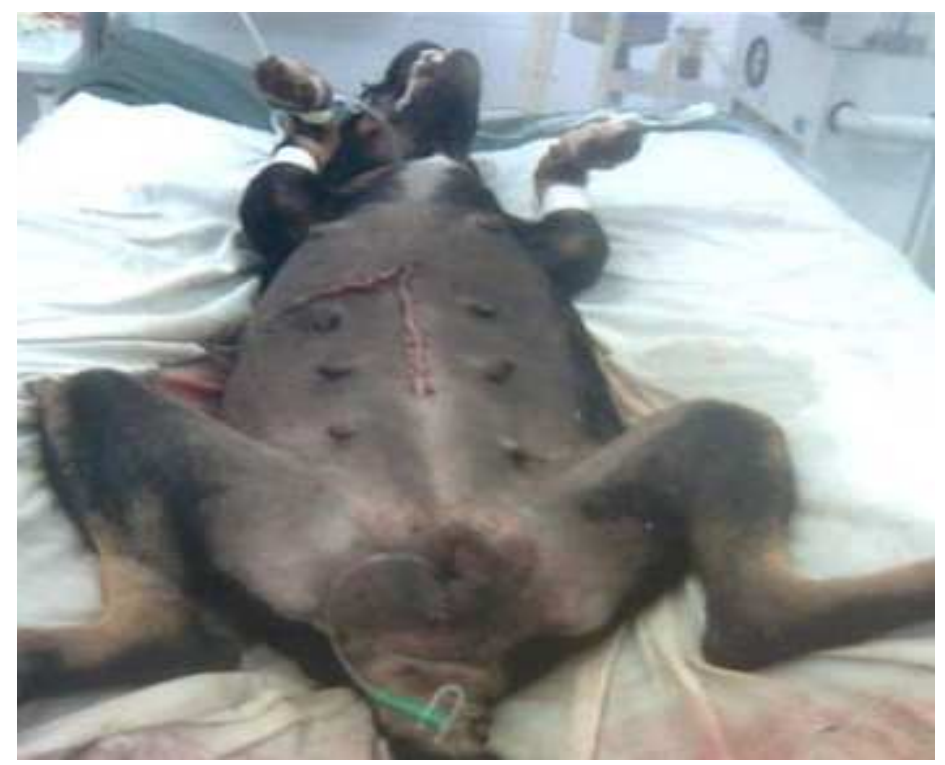

A inspeção renal revelou a presença da larva de D.renale e a presença apenas da cápsula renal no órgão atingido (Figura 5). 
Figura 5- A: Aspecto rim direito após nefrectomia. B: Observação da larva de D.renale e aspecto da cápsula do rim atingido

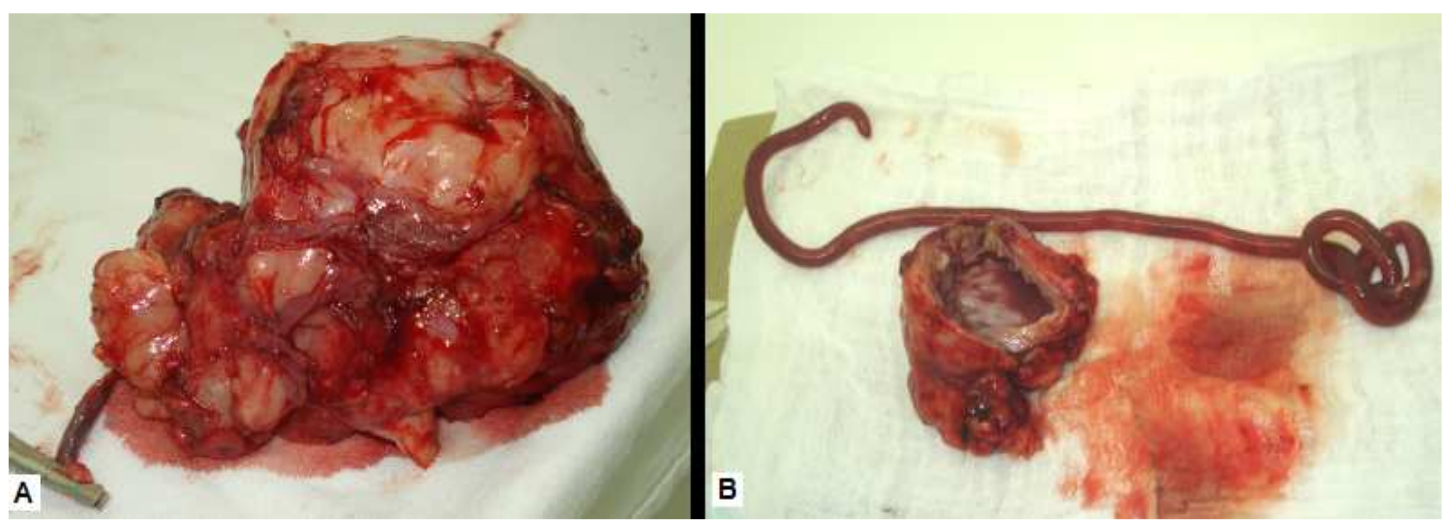

Foi realizado pós operatório com amoxicilina e clavulanato de potássio na dose de $12,5 \mathrm{mg} / \mathrm{kg}$ a cada 12 horas por 15 dias, meloxicam $0,1 \mathrm{mg} / \mathrm{kg}$ a cada 24 horas por 5 dias, tramadol $2 \mathrm{mg} / \mathrm{kg}$ a cada 8 horas e curativos locais nos pontos cirúrgicos, e após sete dias o animal foi liberado para casa, com prescrição de dieta hipercalórica e suplementação com sulfato ferroso. Ao completar trinta dias de pós operatório, foi realizada uma reavaliação do animal, com exames laboratoriais e ultrassonográficos, e este se apresentou em ótimo estado geral, sem alterações aos exames. Foi realizada quimioterapia sugerida por Costa (2009) com sulfato de vincristina na dose de $0,5 \mathrm{mg} / \mathrm{m}^{2}$, a cada sete dias, durante quatro semanas, pois a nodulectomia impossibilitou a ressecção total da massa vaginal. Após a terapia, o animal apresentou remissão total da neoplasia e excelente recuperação por completo.

\section{DISCUSSÃO}

A maioria dos relatos encontrados na literatura, descrevem o parasita em achados de necropsia como Monteiro et al. (2002); Koomers at al. (1999). No presente trabalho pode-se realizar o diagnóstico em tempo viável a conferir uma terapia efetiva ao animal, resultados também obtidos por Ferreira et al., (2010). Observou-se a preferência do D.renale pelo rim direito como na maioria dos trabalhos publicados (FERREIRA NETO et al.,1972; AMATO et al., 1976; SOUZA JUNIOR; PÁDUA, 1977; KOMMERS et al., 1999; MONTEIRO et al., 2002). Anteriormente, acreditava-se que a predominância do rim direito pelo helminto fosse devido às larvas chegarem ao fígado pela corrente sangüínea e passar para o rim devido à proximidade destes. Segundo Osborne et al., (1969), a localização dos parasitas adultos no hospedeiro definitivo parece estar relacionada com o local de penetração das larvas infectantes no trato alimentar. Se as larvas atravessarem a parede gástrica na curvatura menor, podem desenvolver-se entre os lobos do fígado. Quando penetram na curvatura maior, vão para o rim esquerdo. Se penetrarem na parede duodenal, os parasitas adultos desenvolvem-se no rim direito. Esta preferência não está totalmente esclarecida, sendo sugerido por Ferreira Neto et al., (1972) a hipótese do próprio tropismo do parasito levá-lo ao rim direito (COLPO et al 2007). Constatou-se nesse caso, que mesmo o proprietário relatando apatia do paciente, o mesmo o levou apenas por apresentar neoformação vaginal, sendo que o diagnóstico da dioctiofimose foi encontrado nos exames complementares. Apesar de ser considerada uma infecção rara por muitos autores como Forrester (2003), deve-se levar em conta que a ausência de sinais clínicos na maioria dos animais acometidos, pode sugerir que é uma patologia de diagnóstico raro, e não de baixa ocorrência, propondo que ao confirmar o diagnóstico em um paciente, sugere-se a realização de um levantamento na região em que foi confirmado o diagnóstico, como trabalho realizado por Colpo et al., (2007). Corroborando com esta hipótese, a literatura humana ainda constata relatos atuais, sendo 
erronamente diagnosticados como alterações malignas, como demonstra revisão apresentada por Pilscezek (2010), ou relatos de casos como os de Li G et al., (2010) e Ignjatovic I et al., (2003).

Ao questionar os hábitos alimentares do animal em questão, o proprietário relatou apenas a ingestão de alimentos comercial e caseiro, afirmando que o animal não tinha contato com rios, riachos ou lagoas, os quais poderiam servir de fômites para o mesmo, mas, relatou que o animal possuía acesso à rua frequentemente, sugerindo que a infecção possa ter ocorrido de outras formas. Koomers et al. (1999) relataram que dentre essas fontes de infecção alternativas, deve-se considerar que roedores domésticos fazem parte dos hábitos alimentares de cães de rua. Estudos realizados no Japão revelaram o parasitismo por D. renale em ratazanas (Rattus norvegicus) (Taniguchi et al., 1976; Tokiwa et al., 2011). Esses roedores vivem em locais próximos à água, sendo que os esgotos constituem um ambiente onde as ratazanas vivem e se reproduzem com êxito (Silva, 1994). O regime alimentar amplo e a proximidade com a água devem favorecer a infecção de ratazanas, que podem estar envolvidas na rota de infecção de cães de rua, ou em cães semi-domiciliados sendo que para Kommers et al., (1999), a alta ocorrência da doença em cães de rua sugere que a infecção seja relacionada aos hábitos alimentares pouco seletivos desses animais.

A ultrassonografia pode ser um método auxiliar ao diagnóstico, pois geralmente a imagem sugere estruturas como múltiplos anéis com camada hiperecóica e centro hipoecóico (Oliveira et al., 2005), alterações encontradas no presente caso, o que finalizou na realização da urinálise que foi confirmativa para o diagnóstico. Entretanto, deve-se salientar que os ovos serão encontrados no sedimento urinário apenas se a fêmea do parasito está alojada no rim causando infecção (SOLER et al., 2008).

O sucesso alcançado nesse paciente foi obtido devido a eficiência dos exames complementares, meticuloso procedimento cirúrgico e pelo fato do rim esquerdo do paciente ter compensando a ausência da funcionalidade do órgão colateral.

\section{CONCLUSÃO}

Conclui-se que a dioctiofimose quando diagnosticada precocemente resulta em sucesso cirúrgico. Salienta-se a importância da realização de exames complementares de forma rotineira, para que não sejam obtidos diagnósticos incompletos e errôneos.

\section{REFERÊNCIAS}

AMATO, J. F. R.; GRISI, L.; ROSA, V. L. M. da. Reunião dos casos brasileiros de dioctofimose canina com o registro do caso de mais alta intensidade de infecção por DIOCTOPHYMA renale (GOEZE, 1782). Revista Brasileira de Biologia, v. 36, n. 7, p. 117-22, 1976.

BARRIGA, O. Las Enfermedades Parasitarias de los animales domésticos em La América Latina. Santiago: Editorial Germinal, 2002, 247 p.

BROWN, S.A.; PRESTWOOD, A.K. Parasitas do trato urinário. In: KIRK, R.W. Atualização terapêutica veterinária pequenos animais. São Paulo: Manole, 1988. v 2, cap. 13: Distúrbios urinários, p. $1455-1457$.

BRUN, M.V.et al. Nefrectomia laparoscópica em cão parasitado por Dioctiophyma renale- Relato de caso. Arquivos Ciências Veterinárias Zoologia Unipar, v.5, n.1, p.145-152, 2002.

Christie, B.A.; BJORLING, D.E.; In: SLATTER, D. Manual de Cirurgia de Pequenos Animais. $2^{\mathrm{a}}$ ed. v.2. São Paulo: Manole, 1998. p.1698-1713. 
COLPO, C.B.et al. Ocorrência de Dioctophyma renale em cães no município de Uruguaiana-RS. Revista da FZVA. Uruguaiana, v.14, n.2, p. 175-180, 2007.

COSTA, P. R.S.et al. Dioctofimose e leptospirose em um cão - relato de caso. Clínica Veterinária, ano IX, n. 51, p. 48-50, jul./ago. 2004.

COSTA, M.T. Tumor venéreo transmissível canino. In: DALECK, C.R.; DE NARDI, A.B.; RODASKI,S. Oncologia em cães e gatos. São Paulo: Roca, 2009. Cap. 34, p.549.

FERREIRA, V.L.; MEDEIROS, F.B.; JULY, J.B. Dioctophyma renale in a dog: Clinical diagnosis and surgical treatment. Veterinary Parasitology, v.168, p.151-155, 2010.

FERREIRA NETO, J. M.et al. Observações sobre o comportamento do Dioctophyma renale transplantado para a cavidade abdominal do cão. Arquivo da Escola de Medicina Veterinária, v.24, p. 217-219, 1972.

FORRESTER, S. D. Nefropatias e Ureteropatias. In: BIRCHARD, S. J.; SHERDING, R. G. Manual Saunders: clínica de pequenos animais. 2. ed. São Paulo: Roca, 2003. cap. 97, p.1001-28.

FOSSUM, T.W. Cirurgia do rim e ureter. In: FOSSUM, T.W. Cirurgia de Pequenos Animais. 3 ed. Elsevier, 2008. Cap. 24, p.639-640.

GARGILI, A.et al. First case report of Dioctophyma renale (Goeze, 1782) in a dog in Istambul, Turkey.

Turkish Journal of Veterinary and Animal Science, v.26, p.1189-91, 2002.

HEADLUND, C.S. Cirurgia dos sistemas reprodutivo e genital. In: FOSSUM, T.W. Cirurgia de pequenos animais. 3 ed. Elsevier, 2008. Cap. 26, p.709-713.

IGNJATOVIC, I. et al. Infestation of the human kidney with Dioctophyma renale. Urology

Internationalis, v.70, n.1, p.70-3, 2003.

KLEIN, M.K. Tumors of the Female Reproductive System. In: WITHROW, S.J.; VAIL, D.M. Withrow \& MacEwen's Small Animal Clinical Oncology. 4 ed. Missouri: Saunders Elsevier, 2007. Cap. 25, p.615-616.

KOMMERS, G. D.; ILHA, M. R. da S.; BARROS, C. S. L. de. Dioctofimose em cães: 16 casos. Revista Ciência Rural. Santa Maria - RS, v. 29, n. 3, p. 517-522, 1999.

LI, G.et al. Fatal bilateral dioctophymatosis. Journal for Parasitology, v.96, n.6, p.1152-4, 2010.

MONTEIRO, S.G.; SALLIS, E.S.V.; STAINKI, D.R. Infecção natural por trinta e quatro helmintos da espécie Dioctophyma renale (Goeze, 1782) em um cão. Revista da Faculdade de Zootecnia, Veterinária e Agronomia, Uruguaiana, v. 9, n. 1, p. 95-99. 2002.

OLIVEIRA, L.L.et al. 2005. O uso da ultrassonografia para o diagnóstico de Dioctophyma renale em cães: relato de caso. In: CONFERÊNCIA SUL-AMERICANA DE MEDICINA VETERINÁRIA, 5. Annals ... Rio de Janeiro.

OSBORNE, C.A.et al. Dioctophyma renale in the dog. Journal of the American Veterinary Medical Association, v. 155, n. 4, p. 605-619, 1969.

PILSCZEK, F.H. Helminthic infections mimicking malignancy: a review of published case reports. Journal of Infection in Developing Countries, v.4, n.7, p.425-429, 2007.

SILVA, F. Mamíferos silvestres: Rio Grande do Sul. 2 ed. Porto Alegre: Fundação Zoobotânica do Rio Grande do Sul, 1994, p. 184-185. 
SOLER, M.et al. Imaging diagnosis: Dioctophyma renale in a dog. Veterinary Radiology and Ultrasound, v.49, p.307-308, 2008.

SOUZA-JUNIOR, F.L.; PÁDUA, E.B. Dioctophyme renale (Goeze, 1782) (Nematoda, Dioctophymidae) em cães de rua da região de Taubaté (São Paulo, Brasil). Revista de Patologia Tropical, v.6, n.1,2,3,4, p.7-10, 1977.

TANIGUCHI, M.; IKENOUE, S.; SUMITA, N. Discovery of Dioctophyma renale (Goeze, 1782) from a brown rat. Bulletin of the College of Agriculture and Veterinary Medicine Nihon University, v. 33, p. 300-305, 1976.

TOKIWA, T.et al. Dioctophyme renale (Nematoda: Dioctophymatoidea) in the abdominal cavity of Rattus norvegicus in Japan. Parasitology International, v.60, n.3, p. 324-6, 2011.

URQUHART, G.M.et al.Parasitologia Veterinária. 2. ed. Rio de Janeiro: Guanabara Koogan, 1998, p. 86- 87. 